\title{
A Model for the Binding of Fluorescently Labeled Anti-Human CD4 Monoclonal Antibodies to CD4 Receptors on Human Lymphocytes
}

\author{
Lili Wang ${ }^{1}$, Adolfas K. Gaigalas ${ }^{2}$, and Paul C. DeRose ${ }^{1}$ \\ ${ }^{1}$ National Institute of Standards and Technology \\ Gaithersburg, MD 20899, USA \\ ${ }^{2} 2650$ Lake Shore Drive \\ Riviera Beach, FL 33404, USA
}

lili.wang@nist.gov

adolfas.gaigalas@gmail.com

paul.derose@nist.gov

The CD4 glycoprotein is a component of the T cell receptor complex which plays an important role in the human immune response. This manuscript describes the measurement and modeling of the binding of fluorescently labeled anti-human CD4 monoclonal antibodies (mAb; SK3 clone) to CD4 receptors on the surface of human peripheral blood mononuclear cells (PBMC). CD4 mAb fluorescein isothiocyanate (FITC) and CD4 mAb allophycoerythrin (APC) conjugates were obtained from commercial sources. Four binding conditions were performed, each with the same PBMC sample and different CD4 mAb conjugate. Each binding condition consisted of the PBMC sample incubated for $30 \mathrm{~min}$ in labeling solutions containing progressively larger concentrations of the CD4 $\mathrm{mAb}$-label conjugate. After the incubation period, the cells were re-suspended in PBS-based buffer and analyzed using a flow cytometer to measure the mean fluorescence intensity (MFI) of the labeled cell populations. A model was developed to estimate the equilibrium concentration of bound CD4 mAb-label conjugates to CD4 receptors on PBMC. A set of parameters was obtained from the best fit of the model to the measured MFI data and the known number of CD4 receptors on PBMC surface. Divalent and monovalent binding had to be invoked for the APC and FITC CD4 mAb conjugates, respectively. This suggests that the mAb binding depends on the size of the label, which has significant implications for quantitative flow cytometry. The study supports the National Institute of Standards and Technology program to develop quantitative flow cytometry measurements.

Key words: antibody binding; APC; CD4 monoclonal antibody; cooperative binding; FITC; flow cytometry; fluorescence spectroscopy; PBMC.

Accepted: November 7, 2018

Published: December 14, 2018

https://doi.org/10.6028/jres.123.022

\begin{abstract}
Abbreviations
$[\mathrm{A}]$

$\left[\mathrm{A}_{0}\right]$

$\left[\mathrm{A}_{\mathrm{S}}\right]$

$\left[\mathrm{A}_{\mathrm{S}} \mathrm{T}\right]$

concentration of labeled antibody in labeling solution at equilibrium initial concentration of labeled antibody in labeling solution concentration of labeled antibody adsorbed on the surface of the cell concentration of adsorbed labeled antibody bound to single target
\end{abstract}




\begin{tabular}{|c|c|}
\hline$\left[\mathrm{A}_{\mathrm{S}} \mathrm{TT}\right]$ & concentration of adsorbed labeled antibody bound to two targets \\
\hline [AT] & concentration of labeled antibody bound to one target (receptor) \\
\hline$\left[\mathrm{AT}_{\mathrm{m}}\right]$ & concentration of labeled antibody bound to one target on monocytes \\
\hline$[\mathrm{ATT}]$ & concentration of labeled antibody bound to two targets (receptor) \\
\hline$\left[\mathrm{ATT}_{\mathrm{m}}\right]$ & concentration of labeled antibody bound to two targets on monocytes \\
\hline$\left[\mathrm{T}_{0}\right]$ & concentration of targets (receptors) on PBMC \\
\hline$\left[\mathrm{T}_{\mathrm{m} 0}\right]$ & concentration of targets (receptors) on monocytes \\
\hline $\mathrm{ABC}$ & antibodies bound per cell \\
\hline APC & allophycoerythrin, fluorescent protein \\
\hline $\mathrm{Bkg}$ & background signal in flow cytometer measurement \\
\hline CD4 & cluster of differentiation 4 on human T lymphocytes \\
\hline Co1 & company 1 , manufacturer of labeled $\mathrm{mAb}$ \\
\hline $\mathrm{Co} 2$ & company 2, manufacturer of labeled mAb \\
\hline ERF & number of equivalent reference fluorophores \\
\hline $\mathrm{F} / \mathrm{P}$ & average number of fluorophores per protein (mAb) \\
\hline $\mathrm{F}_{\mathrm{ab}}$ & Fab, used in the model to represent F/P \\
\hline FBS & fetal bovine serum \\
\hline FITC & fluorescein isothiocyanate \\
\hline $\mathrm{G}$ & gain of the fluorescence detection system of the flow cytometer \\
\hline $\mathrm{G}_{1}$ & $1 /(\mathrm{mol} / \mathrm{L})$, gain parameter in the fit of model prediction to data \\
\hline$I_{e x}$ & average energy flux of the laser beam in cytometer sensing region \\
\hline $\mathrm{K}_{1}$ & equilibrium dissociation constant for monovalent binding \\
\hline $\mathrm{K}_{2}$ & equilibrium dissociation constant for divalent binding \\
\hline $\mathrm{K}_{\mathrm{D}}$ & protein equilibrium desorption constant \\
\hline $\mathrm{mAb}$ & monoclonal antibody \\
\hline CD4 mAb-APC & CD4 monoclonal antibody APC conjugate \\
\hline CD4 mAb-FITC & CD4 monoclonal antibody FITC conjugate \\
\hline MFI & mean fluorescence intensity, measured \\
\hline $\mathrm{MFI}_{\text {pred }}$ & mean fluorescence intensity, predicted by the model \\
\hline $\mathrm{N}_{\mathrm{ab}}$ & average number of antibodies per PBMC \\
\hline $\mathrm{N}_{\mathrm{at}}$ & average number of antibodies bound to a single target on cell \\
\hline $\mathrm{N}_{\text {att }}$ & average number of antibodies bound to two targets on cell \\
\hline NIST & National Institute of Standards and Technology \\
\hline PBMC & peripheral blood mononuclear cell \\
\hline PBS & phosphate buffered saline buffer \\
\hline QY & fluorescence quantum yield \\
\hline $\mathrm{S}$ & total cell surface area $\left(\mathrm{m}^{2} / \mathrm{L}\right)$ of a cell suspension \\
\hline V450 & trade name of a fluorescent dye \\
\hline
\end{tabular}

\section{Introduction}

The goal of quantitative flow cytometry measurements is to obtain the average number of labeled antibodies bound to specific receptors on the surface of a cell $[1,2]$. The labels conjugated to the antibodies are usually fluorescent dyes, which are excited when the cell passes through the sensing volume of a flow cytometer. The resulting fluorescence emission is detected, and the recorded mean fluorescence intensity (MFI) is an indicator of the average number of labeled antibodies bound per cell (ABC). Flow cytometer detection systems collect real-time fluorescence emission in many wavelength ranges, called fluorescence channels. Each fluorescence channel is dedicated to collecting fluorescence emission from a specific dye conjugated to a specific antibody. Today, many flow cytometers have over 20 fluorescence channels and simultaneously detect many different labeled antibodies bound to different receptors on the surface of the cell [3]. Successful implementation of quantitative flow cytometry would yield a list of the average number of each of the different labeled antibodies bound to the cell. 
The initial step of the quantification process is to calibrate the flow cytometer MFI in terms of the number of equivalent reference fluorophores (ERF) units maintained at the National Institute of Standards and Technology (NIST) [4]. Manufacturers of flow cytometer calibration microspheres will have an opportunity to assign ERF values to their calibration microspheres, providing an international standard with which to calibrate the fluorescence output of all flow cytometers in terms of ERF units. Next, a reference cell, with a known number of CD4 (cluster of differentiation 4 on human T lymphocytes) receptors, will be used to convert the ERF-MFI scale to the ABC-MFI scale, so that an MFI measurement on an analyte cell can be presented as an $\mathrm{ABC}$ value. This second step will require conjugation of different fluorescent labels to the $\mathrm{CD} 4$ antibody so that the conversion to the $\mathrm{ABC}$ scale can be done in all fluorescence channels. Therefore, it is important to examine the binding properties of CD4 antibodies labeled with different fluorescent labels to the CD4 receptor on the surface of the reference cell. Monovalent versus divalent binding, nonspecific binding, and other confounding effects complicate the binding properties of labeled antibodies. Therefore, the production and validation of the reference cell standard require examination of the antibody binding process to ascertain that the binding occurs as expected and that possible confounding effects are taken into account. This article develops a model for the binding of monoclonal antibodies $(\mathrm{mAb})$ to $\mathrm{CD} 4$ receptors on the surface of human lymphocytes, and it uses the model to analyze the binding measurements of the mAb labeled with fluorescein isothiocyanate (FITC), and allophycoerythrin (APC), a fluorescent protein.

\section{Experimental Method}

Cryopreserved normal human peripheral blood mononuclear cells (PBMC; catalog number: CTL-UP1) and anti-aggregate wash supplement (catalog number: CTL-AA-001) were purchased from Cellular Technology (Shaker Heights, OH). ${ }^{1}$ The thawing protocol provided by Cellular Technology for the cryopreserved PBMCs was closely followed using a thaw solution consisting of $1 \mathrm{~mL}$ of anti-aggregate wash supplement and $19 \mathrm{~mL}$ of RPMI-1640 from Invitrogen (Carlsbad, CA). After the thawing procedure, PBMCs in the warm thaw solution were counted using trypan blue staining for an estimation of total cell number. The thawed PBMCs were then centrifuged at $330 \mathrm{~g}$ for $10 \mathrm{~min}$, and the supernatant was discarded. PBMCs were washed once with PBS, pH 7.4, containing $2 \%$ fetal bovine serum (FBS; Sigma-Aldrich, St. Louis, MO), and resuspended in PBS with $2 \%$ FBS to make a final cell concentration of approximately $1 \times$ $10^{7} \mathrm{~mL}^{-1}$. The entire procedure was performed at an ambient room temperature of $22^{\circ} \mathrm{C}$. The thawed PBMC suspension was used for all measurements described below.

\subsection{Staining of PBMC}

A $100 \mu \mathrm{L}$ aliquot of the thawed PBMC suspension (prepared as described above) was added to a defined volume of labeled mAb solution in a test tube prewetted with PBS and $2 \%$ FBS. Table 1 gives the conditions of $\mathrm{mAb}$ solutions used in the staining.

\footnotetext{
${ }^{1}$ Certain commercial equipment, instruments, and materials are identified in this paper to specify adequately the experimental procedure. In no case does such identification imply recommendation or endorsement by the National Institute of Standards and Technology, nor does it imply that the materials or equipment are necessarily the best available for the purpose.
} 
Table 1. Stock solutions of labeled CD4 mAbs used in staining of PBMC.

\begin{tabular}{|c|c|c|c|}
\hline mAb label $^{\mathrm{a}}$ & Concentration & Volume & $\mathrm{F}^{\mathrm{b}}$ \\
\hline CD4 mAb-FITC, Co1 & $3 \mu \mathrm{g} / \mathrm{mL}$ & $2 \mathrm{~mL}$ & 4.21 \\
\hline CD4 mAb-APC, Co1 & $6 \mu \mathrm{g} / \mathrm{mL}$ & $0.5 \mathrm{~mL}$ & 1.30 \\
\hline CD4 mAb-FITC, Co2 & $100 \mu \mathrm{g} / \mathrm{mL}$ & $0.5 \mathrm{~mL}$ & 6.1 \\
\hline CD4 mAb-APC, Co2 & $50 \mu \mathrm{g} / \mathrm{mL}$ & $0.5 \mathrm{~mL}$ & 1.4 \\
\hline
\end{tabular}

${ }^{\mathrm{a}} \mathrm{Co} 1$ = company source $1, \mathrm{Co} 2=$ company source 2 .

${ }^{\mathrm{b}} \mathrm{F} / \mathrm{P}=$ average number of fluorophores per protein $(\mathrm{mAb})$.

The suspensions of labeled $\mathrm{mAb}$ and cells were vortexed and then incubated for $30 \mathrm{~min}$ in the dark at room temperature $\left(22^{\circ} \mathrm{C}\right)$ without further mixing. After the $30 \mathrm{~min}$ incubation time, the cells were washed with $2 \mathrm{~mL}$ of the PBS and $2 \%$ FBS buffer and centrifuged, and the stained cells were re-suspended in $0.5 \mathrm{~mL}$ of the same buffer. To construct a titration curve for the labeled mAbs, the starting point was the amount of antibody recommended by the manufacturer to obtain saturation. Approximately eight 2 -fold dilutions and one 1.5-fold increase of the recommended amount of the antibody were included in the titration curve. The stained PBMCs were analyzed using an Aria II flow cytometer within $2 \mathrm{~h}$ after the preparation of the sample. The FITC and APC labels were measured using the FITC and APC fluorescence channels of the Aria II flow cytometer.

\subsection{Measurement of the Concentration of CD4+ Lymphocytes}

To determine accurate concentrations of CD4+ lymphocytes present in the staining tubes, at least two extra thawed PBMC staining tubes were used as described in Sec. 2.1. The concentration of PBMCs in the staining tube was measured relative to the microsphere count standard in a TruCount tube (BD Biosciences, San Jose, CA). The concentration measurement was performed by adding $100 \mu \mathrm{L}$ of the thawed PBMC suspension (the same suspension used in the staining tubes discussed in Sec. 2.1) and a defined volume of labeled $\mathrm{mAb}$ to a TruCount tube. The labeled $\mathrm{mAb}$ concentration was chosen to ensure saturating condition for the staining of PBMC in the TruCount tube. In order to minimize the loss of cells, a no-wash procedure was performed after sample staining. A total final volume of $1 \mathrm{~mL}$ of stained PBMCs in the TruCount tube was made with PBS and $2 \%$ FBS. The stained samples were analyzed using the Aria II flow cytometer within $2 \mathrm{~h}$ after sample staining. Moreover, the gating of $\mathrm{CD} 4+$ lymphocytes was also confirmed by staining of both CD3 V450 and CD4 FITC or APC in some cases. The concentration of CD4+ lymphocytes in the TruCount tube was determined to be $0.2 \times 10^{6}$ cells $/ \mathrm{mL}$. After adjusting for the 10 -fold dilution, the concentration of CD4+ cells in TruCount tubes during cell staining was approximately $0.2 \times 10^{7}$ cells $/ \mathrm{mL}$.

\section{Model of the Binding of Labeled CD4 mAbs to CD4 Receptors}

The model is based on a sequence of binding events shown in Fig. 1 [5]. 


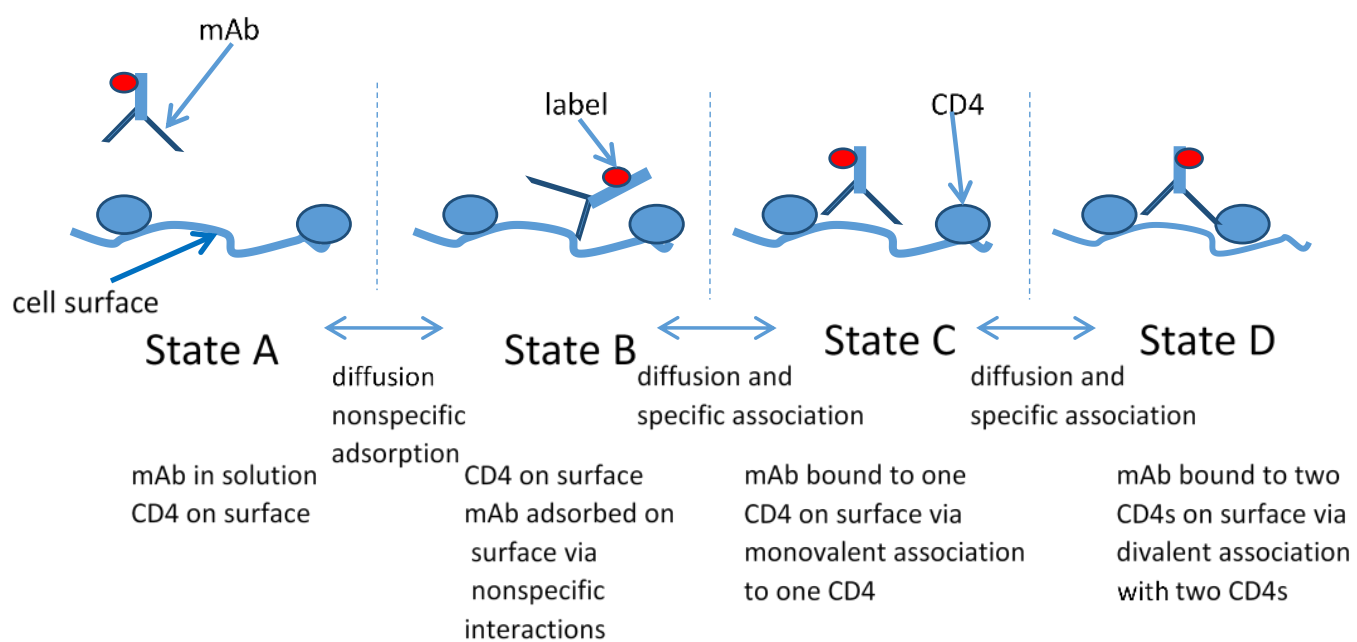

Fig. 1. The dashed vertical lines separate four states that model the binding of a labeled CD4 mAb to a CD4 receptor on the surface of a cell. The small red circle is the label, the "Y" shape is the CD4 mAb, the large blue circles are the CD4 receptors, and the horizontal wavy line is the surface of the cell. In state $\mathrm{A}$, the $\mathrm{CD} 4 \mathrm{mAb}$ is in solution, and the CD4 is on the surface. In state $\mathrm{B}$, the CD4 mAb is associated with the cell surface. In state $\mathrm{C}$, the $\mathrm{CD} 4 \mathrm{mAb}$ has undergone a monovalent binding to the CD4 receptor on the surface. In state $\mathrm{D}$, the $\mathrm{CD} 4 \mathrm{mAb}$ is bound to two (divalent) $\mathrm{CD} 4$ receptors. The model assumes that the $\mathrm{CD} 4 \mathrm{mAb}$ binding is a sequential process going from state A to state D.

The process of binding may consist of four identifiable states. State A consists of the labeled CD4 $\mathrm{mAb}$ in solution and CD4 receptor on the cell surface. State B consists of labeled CD4 mAb associated with the surface of the cell. The association is via nonspecific interactions between ionic and hydrophobic complementary sites on the labeled CD4 mAb and the cell surface. The nonspecific adsorption can lead to a significant population of labeled CD4 mAb in state $\mathrm{B}$. In state $\mathrm{C}$, the labeled CD4 mAb has undergone a monovalent binding to one CD4 receptor. Finally, in state D, the labeled CD4 mAb is bound to two CD4 receptors via a divalent bond. The transition from state $A$ to state $B$ should consider the roughness of the cell surface. This transition is expected to be diffusion controlled, and diffusion near a surface is influenced by the roughness of the surface. The model is based on a fundamental assumption that the transition from states A to D is sequential.

Section 3.1 describes a simplified model that neglects state B and jumps directly from state A to state C. Binding measurements by themselves do not provide enough information to identify the steps in the transition from state $\mathrm{A}$ to state $\mathrm{C}$. Therefore, the model uses a phenomenological approach and defines a dissociation equilibrium constant that describes the combined result of all steps in the path between states A and C. In Sec. 6 of the manuscript, we try to make it plausible that the likely path from A to C goes through state B, which is populated via nonspecific adsorption. During lymphocyte labeling, there is a substantial population of monocytes that also have CD4 receptors. Section 5 describes a modification of the model to include binding of CD4 mAb to lymphocytes in the presence of monocytes.

\subsection{A Simplified Model for Lymphocyte Labeling}

The model is implemented by writing the equilibrium relation for the transitions from $\mathrm{A}$ to $\mathrm{C}$ and from C to D. These are given by Eq. (1) and Eq. (2).

$$
\begin{gathered}
0=k_{+1}[A][T]-k_{-1}[A T]-k_{+2}[A T][T]+k_{-2}[A T T] . \\
0=k_{+2}[A T][T]-k_{-2}[A T T] .
\end{gathered}
$$


Here, $[A]$ is the volume concentration of labeled CD4 mAb in the labeling suspension, and $[T]$ is the apparent volume concentration of CD4 receptors on cell surfaces. The association rate constant, $\mathrm{k}_{+1}$, describes the transition from A to $\mathrm{C}$. It depends on the diffusive flux of labeled $\mathrm{mAb}$ to the receptor CD4 and the sequence of orientation changes resulting in the monovalent binding of the labeled CD4 mAb and the CD4 receptor. The bound system exchanges energy with the surrounding fluid, resulting in dissociation characterized by a rate constant $\mathrm{k}_{-1}$. In state $\mathrm{C}$, the labeled $\mathrm{CD} 4 \mathrm{mAb}$ may be oriented for optimal association with another $\mathrm{CD} 4$ receptor molecule. In addition, the first binding locates the labeled CD4 mAb on the cell surface, where the local concentration of CD4 is much higher than the average concentration in suspension. Therefore, the first binding may increase the likelihood of the second binding and result in an association constant $\mathrm{k}_{+2}$ much larger than $\mathrm{k}_{+1}$. Such behavior has been investigated recently Ref. $[6,7,8]$. The transition from state $\mathrm{C}$ to $\mathrm{D}$ could appear like cooperative binding of monovalently bound CD4 mAb to another CD4 receptor. The relation between the apparent volume concentration and the number of receptors on the cell surface is given by Eq. (3).

$$
\left[T_{0}\right]=N_{\text {cells }} * N_{C D 4} * \frac{1000}{N_{A}}, \mathrm{~mol} / \mathrm{L},
$$

where $N_{\text {cells }}$ is the number of cells per milliliter of labeling suspension, $N_{\mathrm{CD} 4}$ is the average number of CD4 receptors per cell, and $N_{A}$ is Avogadro's number. The volume concentration of the CD4 receptors is very inhomogeneous since they are concentrated on cell surfaces. This fact has to be kept in mind when discussing the meaning of the rate constants in Eq. (1) and Eq. (2). $\left[T_{0}\right]$ is the initial concentration of unbound receptors, and $\left[A_{0}\right]$ is the initial concentration of labeled $\mathrm{CD} 4 \mathrm{mAb}$ at the start of the labeling process. The value of $\left[A_{0}\right]$ was freely adjusted, while $\left[T_{0}\right]$, which depends on the known number of cells in the labeling suspension and the average number of CD4 receptors on a single cell, was a fit parameter. The symbols $[A T]$ and $[A T T]$ represent the apparent volume concentrations of labeled CD4 mAb bound to the receptor via monovalent and divalent binding, respectively. The actual number of bound receptors on a cell can be found by inverting Eq. (3). Since the binding between an antibody and its antigen is reversible and preserves the molecular identity of molecules involved in the binding, we can write relations for the conservation of the number of molecules given in Eq. (4) and Eq. (5).

$$
\begin{gathered}
{\left[T_{0}\right]=[T]+[A T]+2 *[A T T] .} \\
{\left[A_{0}\right]=[A]+[A T]+[A T T] .}
\end{gathered}
$$

The concentrations of monovalently and divalently bound receptors, $[A T]$ and $[A T T]$, respectively, are zero at the start of the incubation. Equations (1), (2), (4), and (5) represent the model. We are interested in the values $[A T]$ and $[A T T]$, since their sum is proportional to the mean fluorescence signal (MFI) measured in a flow cytometer. The other quantities that were measured are $N_{\text {cells }}$ and $\left[A_{0}\right]$. Equation (2) and Eq. (4) were used to solve for $[A T T]$ in terms of $[A T]$, with the result shown in Eq. (6).

$$
[A T T]=\frac{[A T] *\left(\left[T_{0}\right]-[A T]\right)}{K_{2}+2 *[A T]} .
$$

The dissociation equilibrium constants $K_{1}$ and $K_{2}$ are defined as $K_{1}=k_{-1} / k_{+1}$ and $K_{2}=k_{-2} / k_{+2}$, respectively. The expected MFI is proportional to the total number of labels on the cell and is given by Eq. (7).

$$
M F I \propto[A T]+[A T T]=\frac{[A T] *\left(K_{2}+\left[T_{0}\right]\right)+[A T]^{2}}{K_{2}+2 *[A T]} .
$$


[AT] was found by first adding Eq. (1) and Eq. (2) to obtain $K_{2} \times[\mathrm{AT}]=[\mathrm{A}] \times[\mathrm{T}]$. Then, Eqs. (4), (5), and (6) were used to get an equation containing only [AT]. The equation was further simplified to a third-order polynomial in [AT], as shown in Eq. (8),

$$
c_{0}+c_{1} *[A T]+c_{2} *[A T]^{2}+c_{3}[A T]^{3}=0,
$$

where the coefficients are given by:

$$
\begin{aligned}
& c_{0}=\left[A_{0}\right] *\left[T_{0}\right] . \\
& c_{1}=-\left(\left[A_{0}\right]+\left[T_{0}\right]+K_{1}-\frac{2 *\left[A_{0}\right] *\left[T_{0}\right]-\left[T_{0}\right]^{2}}{K_{2}}\right) . \\
& c_{2}=1-\frac{2 *\left[A_{0}\right]+4 * K_{1}}{K_{2}} . \\
& c_{3}=\frac{K_{2}-4 * K_{1}}{K_{2}{ }^{2}} .
\end{aligned}
$$

The model binding curve was obtained by finding the roots of Eq. (8) as a function of the initial concentration of labeled CD4 mAb, given by $\left[A_{0}\right]$ in Eq. (8). The roots were found using polyroot function in Mathcad 15. Only the physically meaningful roots were considered, where both $[A T T]$ and $[A T]$ are real positive numbers and approached zero as $\left[A_{0}\right]$ approached zero. In addition, the predicted MFI at equilibrium had to be associated with a value of $\left[T_{0}\right]$ approximately equal to $145,000 \pm 9000$, the previously measured number of CD4 receptors on the surface of normal lymphocytes $[9,10]$. The value of $\left[T_{0}\right]$ was not constrained during the fit of the model to the observed MFI values. However, if the fit was consistent with several roots, then the root that gave a value of $\left[T_{0}\right]$ closest to 145,000 was chosen. It was observed that root 1 corresponded to physically meaningful binding curves when $K_{1}$ was smaller than $K_{2}$. This case is illustrated in Fig. 2(a), where the dotted trace is the concentration of monovalently bound labeled CD4 $\mathrm{mAb},[\mathrm{AT}]$, and the dashed trace is the concentration of divalently bound labeled CD4 mAb, [ATT]. The solid trace is the sum of the two concentrations. This is the result one would expect for the case where the binding of the first CD4 receptor and the binding of the second CD4 receptor to the CD4 mAb were independent of each other. The second root of Eq. (8) gave physically meaningful results when $K_{2}$ was much smaller than $K_{1}$. This case is illustrated in Fig. 2(b), with the same notation of the traces as in Fig. 2(a). The binding curves for the two cases are very different. In Fig. 2(a), monovalent binding dominates, and at equilibrium, there is almost zero contribution from divalent binding. In other words, there is a single labeled CD4 mAb for each CD4 receptor. In Fig. 2(b), divalent binding dominates at equilibrium, with a small contribution from monovalent binding. In this case, there is approximately one labeled CD4 mAb for every two CD4 receptors. Mathcad was used for the initial exploration of the roots of the third-order polynomial in Eq. (8), and the results are shown in Figs. 2(a) and 2(b). 
(a)

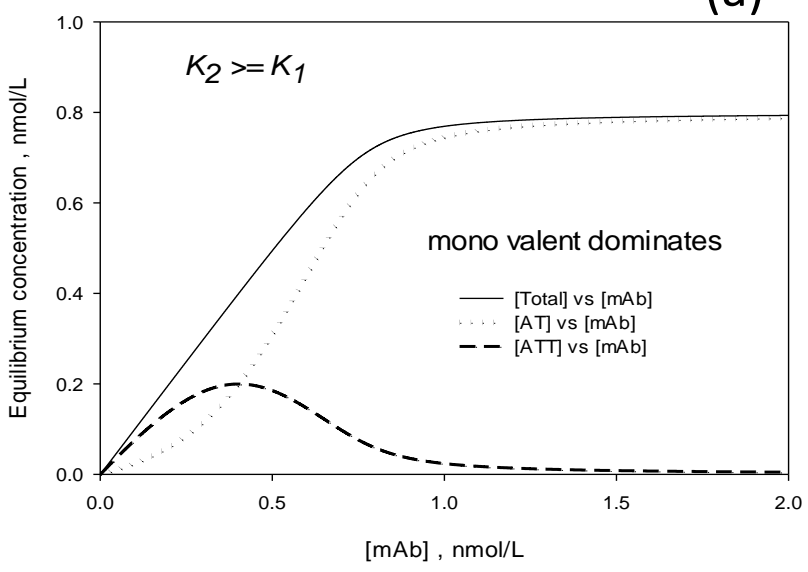

(b)

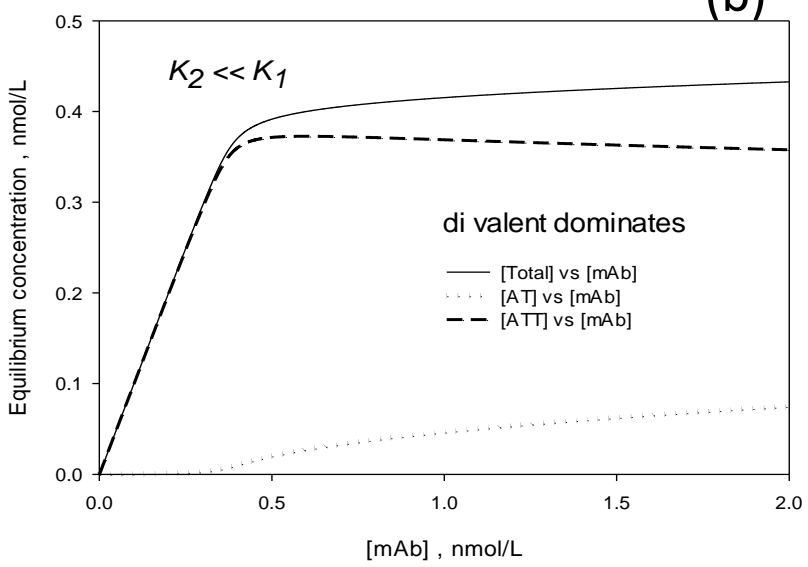

Fig. 2. (a) The solid line gives the prediction of the equilibrium concentration of surface-bound mAb for the case where the divalent dissociation constant $K_{2}$ is larger than the dissociation constant, $K_{1}$, for monovalent binding. The horizontal axis gives the initial concentration of $\mathrm{mAb}$ in the labeling suspension. The dotted line and the dashed line give the predicted concentration of monovalently bound $\mathrm{CD} 4 \mathrm{mAb}$, [AT], and divalently bound CD4 mAb, [ATT], respectively. At saturation, monovalent binding dominates. The concentrations can be related to the number of mAbs on the cell surface using Eq. (3) in the text. The concentration of CD4 receptors was set to $0.8 \mathrm{nmol} / \mathrm{L}$. (b) The meaning of the lines is the same as in (a); the only difference is that $K_{2} \ll<K_{1}$. At saturation, divalent binding dominates. The predictions were calculated using Eq. (8) in the text. The solid lines in (a) and (b) correspond to different roots of Eq. (8).

The analysis of the binding curves and the model calculations were performed using Matlab. Equations (1), (2), (4), and (5) were solved numerically using the Matlab function $f$ solve, and the fit to the measured binding curve was performed using the Matlab function lsqnonlin. The details of the analysis are described in the Appendix. The numeric solution was identical to that given by Eq. 8 .

In summary, the model assumed the existence of three states for the labeled CD4 mAb and the CD4 receptors. The first state corresponds to unassociated labeled CD4 mAb and CD4 receptors, the second state involves an association between a single labeled CD4 mAb and a single CD4 receptor, and a third state is where each labeled CD4 mAb is associated with two CD4 receptors. The model predicts the equilibrium concentrations of all four species ([A], $[T],[A T]$, and $[A T T])$. The four concentrations depend on the set of parameters, $K_{1}, K_{2},\left[A_{0}\right]$, and $\left[T_{0}\right]$. By varying the initial concentration of labeled CD4 mAb, $\left[A_{0}\right]$, in the labeling solution, it was possible to obtain a set of concentrations $[A],[T],[A T]$, and $[A T T]$ for each value of $\left[A_{0}\right]$. The value of $[A T]+[A T T]$ is directly proportional to the value of MFI measured by a flow cytometer. A model binding curve was obtained by plotting the predicted values of MFI as a function 
of $\left[A_{0}\right]$. The set of parameters, $K_{1}, K_{2}$, and $\left[T_{0}\right]$, was obtained by finding the best fit between the measured values of MFI and those predicted by the model. It should be emphasized that the model predictions for MFI are based on the values of $\left[A_{0}\right]$, and some general assumptions inherent in the model that describe how $\left[A_{0}\right]$ is apportioned among the states $\mathrm{A}, \mathrm{C}$, and $\mathrm{D}$ with concentrations [A], [AT], and [ATT], respectively. The solid lines in Figs. 3(a) to 3(d) show the best fits to data, represented by the solid circles. The figures will be discussed in the section below.

\section{Results and Discussion}

When the cell passes through the laser beam in a flow cytometer, the cell emits fluorescence from excited fluorophores conjugated to antibodies bound to the receptors on the cell. The fluorescence is collected and then directed to a set of selection filters, which partition the fluorescence into various wavelength ranges (called fluorescence channels). Each wavelength range is sent to an individual photomultiplier detector. The output of each photomultiplier detector is processed and converted into a digital number representing the intensity of the emitted fluorescence. The flow cytometer collects the output from many cells of the same type and provides a histogram of the distribution of fluorescence pulse amplitudes from the cells. The mean fluorescence intensity (MFI) is modeled in Eq. (9), where the various terms characterize each of the steps in the detection process.

$$
M F I=\Gamma G I_{e x}(\Delta t) P \sigma_{e x}(Q Y) F_{a b} N_{a b} .
$$

The first five factors in Eq. (9) characterize the flow cytometer, while the last four factors characterize the cells with the labeled mAb attached to the receptors on the cell surface. The symbol $\Gamma$ represents the throughput of the collection optics and the filter wheel. The fluorescence from the cell is emitted in all directions, and the collection optics collect a fraction of the emitted fluorescence. The collected fluorescence is then passed through a series of optical filters to select the fluorescence wavelengths characteristic of the label. The filters define a fluorescence channel on the flow cytometer. The symbol $G$ is the total gain, which is composed of the gain of the photomultiplier detector and the gain of the electronic components that further amplify the photomultiplier detector output. $I_{e x}$ gives the average energy flux of the laser beam at the point where the laser beam intersects the streaming cells, and $\Delta t$ is the average time spent by the cell in the laser beam. The symbol $P$ in Eq. (9) is a measure of the effect of laser polarization on the magnitude of the response. Usually, the illuminating laser beam is linearly polarized in the direction perpendicular to the plane defined by the laser propagation direction and the direction of the detection cone. This maximizes the response from labels attached to microspheres or cells and excited by the polarized laser beam. (The fluorescence lifetime is usually less than $10 \mathrm{~ns}$, and the initial orientation of the transition dipole moments of the immobilized labels does not randomize significantly.) The effect of laser polarization is difficult to quantify, since the details of the illumination and detector design are proprietary information. The assumption is made that once the instrument is set up, the factor $P$ stays constant for the duration of the measurements. The next four factors in Eq. (9) describe the properties of the fluorescent labels. The symbol $\sigma_{e x}$ is the absorption cross section at the laser wavelength, and together with $I_{e x}$ and $\Delta t$, it gives the amount of laser light absorbed by one label on the cell. $Q Y$ is the fluorescence quantum yield of the label on the cell surface, and it gives the probability that after the label absorbs a photon, a fluorescence photon will be emitted. $F_{a b}$ gives the average number of labels conjugated to the mAb. The $Q Y$ of the labels on the antibodies bound to cells is difficult to measure. The product $Q Y \times F_{a b}$ in Eq. (9) can be written as $Q Y / Q Y_{\text {ref }} \times Q Y_{\text {ref }} F_{a b}$, where $Q Y_{\text {ref }}$ is the known quantum yield of the reference solution of the labeling fluorophores. The relative quantum yield, $Q Y / Q Y_{\text {ref }}$, can be estimated using fluorometer steady-state measurements or lifetime measurements. $N_{a b}$ is the average number of labeled mAb bound to a lymphocyte cell. The value of $N_{a b}$ is the end result of the labeling process and is the object of the modeling described above. $F_{a b}$ is determined by the process used to create the $\mathrm{mAb}$ and conjugate labels onto the cell. In order 
to characterize the response of the flow cytometer to cells with a given label, all of the factors in Eq. (9) have to be considered. An important parameter that is not shown in Eq. (9) is the background, Bkg, of the MFI signal. The background subtraction modifies the MFI data so that the subtracted MFI values approach zero when the labeled $\mathrm{mAb}$ concentration in the labeling solution approaches zero. Inclusion of this parameter is justified physically, and it is likely due to a combination of electronic noise, scattered light, and autofluorescence.

\subsection{MFI and the Prediction of the Model}

The solid circles in Figures 3(a) to 3(d) show the measured MFI (vertical axis) for cells labeled with a solution containing labeled $\mathrm{CD} 4 \mathrm{mAb}$ for which the initial concentration is given on the horizontal axis. The solid trace through the solid circles gives the value of MFI predicted by the model given by the sum of [ATT] and [AT] and the background parameter, Bkg. The inset plot in each of the figures gives the model prediction of the MFI versus the actual number of monovalently $\left(N_{\mathrm{at}}\right)$ and divalently $\left(N_{\text {att }}\right)$ labeled CD4 mAb bound to CD4 receptors on the surface of the cell. The values of $N_{\mathrm{at}}+N_{\mathrm{att}}$ and [AT] + [ATT] are related by Eq. (3), discussed previously.
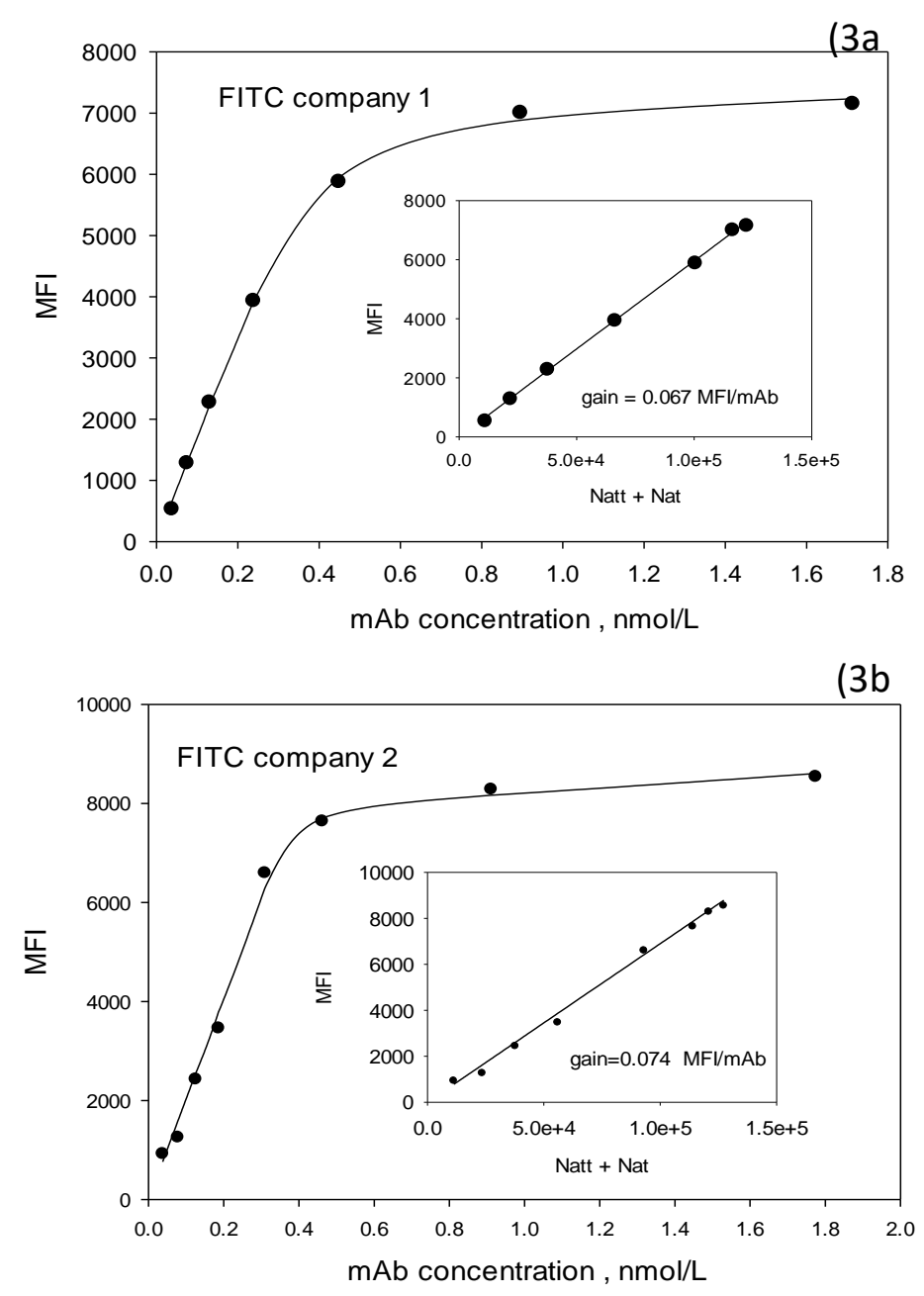

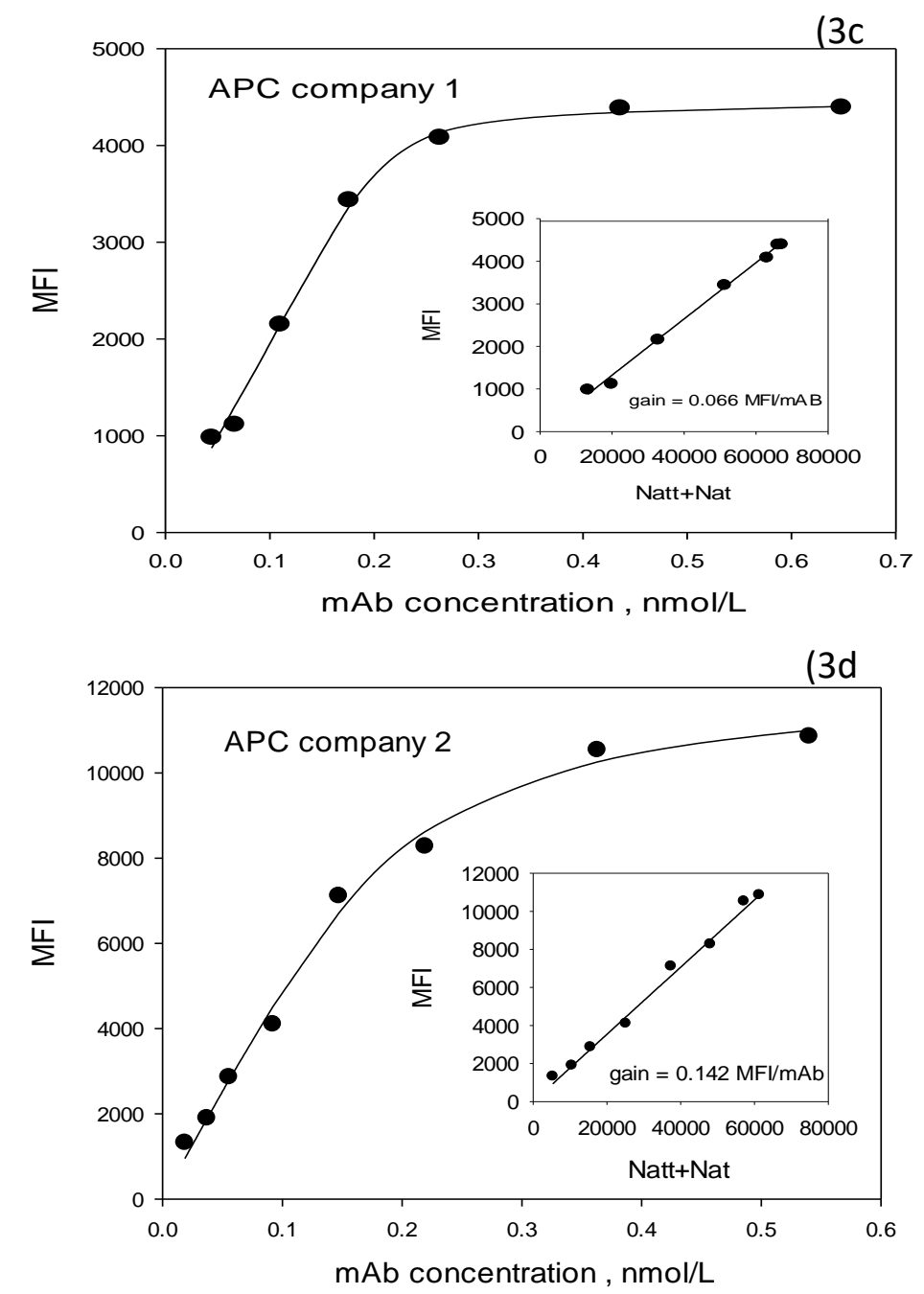

Fig. 3. (a) The solid circles give the measured mean fluorescence intensity (MFI) associated with peripheral blood mononuclear cells (PBMC) labeled with solutions containing different concentrations of CD4 mAb-FITC conjugates provided by company 1 . The solid line is the prediction of the model given in detail in Table 2 using parameters shown in the first row of Table 3 . The monovalent binding dominated at saturation. The inset plot shows the dependence of MFI on the total number of mAbs bound to the cell surface at equilibrium, $N_{\text {att }}+N_{\text {at. }}$ As expected, the dependence is linear, and the slope of the line, 0.067, gives the label gain in units of $\mathrm{MFI} / \mathrm{mAb}$. (b) Identical information as described in part (a), except the CD4 mAb-FITC conjugates were provided by company 2. The parameters used to generate the solid curve are given in row 3 of Table 3 . The label gain was $0.074 \mathrm{MFI} / \mathrm{mAb}$, and monovalent binding dominates. (c) Identical information as described in part (a), except the CD4 mAb-APC conjugates were provided by company 1 . The parameters used to generate the solid curve are given in row 5 of Table 3 . The label gain was $0.066 \mathrm{MFI} / \mathrm{mAb}$, and divalent binding dominates. (d) Identical information as described in part (a), except the CD4 mAb-APC conjugates were provided by company 2. The parameters used to generate the solid curve are given in row 7 of Table 3 . The label gain was $0.142 \mathrm{MFI} / \mathrm{mAb}$, and divalent binding dominates.

Below is a detailed discussion of the generation of Fig. 3(a). The same procedure was followed to generate the other figures, Figs. 3(b), 3(c), and 3(d). 


\subsubsection{Analysis of Cells Labeled with CD4 mAb-FITC Conjugates}

The first column of Table 2 gives the initial CD4 mAb-FITC concentrations used in labeling of the cells, and the second column gives the measured MFI. The third column gives the predicted $M F I_{\text {pred }}$ values. The predictions were based on Eq. (9), with $M F I_{\text {pred }}=G_{\mathrm{I}} \times([A T]+[A T T])+$ Bkg. The parameter $\mathrm{G}_{\mathrm{I}}$ represents the product of all the factors in Eq. (9) multiplying $N_{\mathrm{ab}}$, and a conversion factor relating $N_{\mathrm{ab}}$ to $[A T]+[A T T]$ (see Eq. (3)). The sum, $[A T]+[A T T]$, was obtained from a solution of Eqs. (1), (2), (4), and (5) with a given set of parameter values. The best $M F I$ prediction was obtained by requiring that the sum $\left(M F I_{\text {pred }}-M F I\right)^{2}$ evaluated at all concentrations of CD4 mAb-FITC was a minimum. The search for a minimum was performed using the Matlab function lsqnonlin, which varied the parameters. The fourth and fifth columns in Table 2 give the predicted contributions from CD4 mAb-FITC with monovalent and divalent binding to CD4 receptors. The sixth column gives the total number of bound CD4 mAb-FITC receptors on the cell obtained from the sum $[A T]+[A T T]$. Columns 1 and 2 were used to generate the plot of the solid circles, and columns 1 and 3 were used to generate the solid trace in Fig. 3(a). The inset plot in Fig. 3(a) was obtained from column 2 and column 6 of Table 2. The inset plot in Fig. 3(a) is a good summary of the model results. The straight line shows that the values of MFI depend linearly on the total number of CD4 mAb-FITC receptors on the cell given by $N_{\text {at }}+N_{\text {att }}$. The points on the straight line converge to a limiting value for large values of $N_{\text {at }}+N_{\text {att }}$, indicating the onset of saturation of binding.

Table 2. Concentration of the labeling solution of CD4 mAb-FITC, Co1, and the measured MFI. The last four columns show the predictions of the best fit to the MFI data.

\begin{tabular}{|c|c|c|c|c|c|}
\hline $\begin{array}{c}\text { Concentration } \\
(\mathrm{nmol} / \mathrm{L})\end{array}$ & MFI & $G_{\mathrm{I}} \times(\mathrm{AT}+\mathrm{ATT})+\mathrm{Bkg}$ & $G_{\mathrm{I}} \times \mathrm{AT}$ & $G_{\mathrm{I}} \times \mathrm{ATT}$ & $N_{\text {AT }}+N_{\text {ATT }}$ \\
\hline 0.0374 & 563 & 675 & 675 & 0.027 & 10145 \\
\hline 0.0744 & 1313 & 1329 & 1328 & 0.0483 & 19970 \\
\hline 0.13 & 2306 & 2276 & 2276 & 0.0698 & 34210 \\
\hline 0.238 & 3968 & 3933 & 3932 & 0.0819 & 59104 \\
\hline 0.448 & 5912 & 5962 & 5961 & 0.0522 & 89595 \\
\hline 0.895 & 7039 & 6961 & 6961 & 0.0196 & 104616 \\
\hline 1.713 & 7186 & 7235 & 7235 & 0.0086 & 108725 \\
\hline
\end{tabular}

The first row of Table 3 gives the values of the parameters that gave the best fit to the solid circles in Fig. 3(a) and generated the predictions shown in Table 2. Rows 3, 5, and 7 in Table 3 hold the parameter values that gave best fits to the data obtained with other labels, the names of which are shown in column 2 of Table 3. Rows 2, 4, 6, and 8 of Table 3 contain the estimates of uncertainties obtained from the fit. As stated previously, the parameters $G_{\mathrm{I}}$ and Bkg were estimated together with parameters $K_{1}, K_{2}$, and [ $\left.T_{0}\right]$ by requiring the minimum deviation between the values of MFI and the values of $\left(G_{\mathrm{I}} \times[\mathrm{AT}+\mathrm{ATT}]+\mathrm{Bkg}\right)$. The parameter $\left[T_{0}\right]=0.37 \mathrm{nmol} / \mathrm{L}$ in row 1 of Table 3 implies a total of 112,000 receptors on the cell, which is slightly greater than the value of 109,000 in column 6 of Table 2 at the highest concentration of CD4 mAb-FITC in the labeling solution. This may indicate a small departure from saturation at the highest concentration of CD4 mAb-FITC in the labeling solution. Similar results were obtained for CD4 mAbFITC from company 2, shown in Fig. 3(b). The best-fit parameters are shown in row 3 of Table 3 . The total number of CD4 receptors obtained from the parameter $\left[T_{0}\right]$ was 116,000 , which is consistent with the results for CD4 mAb-FITC from company 1. 
Table 3. The model parameters that gave the best fit to the MFI data.

\begin{tabular}{|c|c|c|c|c|c|c|c|c|c|}
\hline & $\begin{array}{c}\text { Label on } \\
\mathrm{mAb}\end{array}$ & $K_{1}$ & $K_{2}$ & $\begin{array}{c}{\left[T_{\mathrm{o}}\right],} \\
\mathrm{nmol} / \mathrm{L}\end{array}$ & $B k g$ & $\begin{array}{c}G, \\
\mathrm{MFI} / \mathrm{nmol}\end{array}$ & $\begin{array}{c}\text { Label gain } \\
\mathrm{MFI} / \mathrm{mAb}\end{array}$ & $\begin{array}{c}\text { Total CD4 } \\
\text { on cell }\end{array}$ & Binding \\
\hline 1 & FITC-Co1 & $3.71 * 10^{-2}$ & $8.39^{*} 10^{+3}$ & 0.371 & 0 & 20038 & 0.067 & 111700 & monovalent \\
\hline 2 & Std. Dev. & $3.6^{*} 10^{-3}$ & $3.19 * 10^{+4}$ & 0.004 & 43 & 180 & 0.001 & 1180 & \\
\hline 3 & FITC-Co2 & $7.60^{*} 10^{-3}$ & $5.52^{*} 10^{+4}$ & 0.386 & 677 & 22590 & 0.074 & 116300 & monovalent \\
\hline 4 & Std. Dev. & $6.1 * 10^{-3}$ & $4.5^{*} 10^{+5}$ & 0.014 & 180 & 670 & 0.003 & 4200 & \\
\hline 5 & APC-Co1 & $7.00^{*} 10^{+5}$ & $7.21^{*} 10^{-10}$ & 0.463 & 3760 & 19960 & 0.066 & 139400 & divalent \\
\hline 6 & Std. Dev. & $4.94 * 10^{+5}$ & $1.06 * 10^{-9}$ & 0.011 & 65 & 390 & 0.002 & 3240 & \\
\hline 7 & APC-Co2 & $5.99 * 10^{+6}$ & $3.69 * 10^{-10}$ & 0.527 & 555 & 42200 & 0.142 & 159000 & divalent \\
\hline 8 & Std. Dev. & $4.09 * 10^{+6}$ & $6.4 * 10^{-10}$ & 0.022 & 219 & 1500 & 0.007 & 6800 & \\
\hline
\end{tabular}

\subsubsection{Analysis of CD4 mAb-APC Conjugates}

Applying the same analysis (monovalent binding with $K_{1}<<K_{2}$ ) to CD4 mAb labeled with APC lead to problems. This is illustrated for the case of CD4 mAb-APC from company 2 . The fit to the data was good and almost indistinguishable from that shown in Fig. 3(d) (to be discussed later). However, the parameter $\left[T_{0}\right]=0.217 \mathrm{nmol} / \mathrm{L}$ gave $65,000 \pm 6000 \mathrm{CD} 4$ receptors on the lymphocyte. This value is inconsistent with the results obtained from the analysis of CD4 mAb-FITC conjugates from the two companies. Furthermore, it is also inconsistent with a recently published value of 145,000 $\pm 9000 \mathrm{CD} 4$ receptors on T lymphocytes $[9,10]$. Possible sources of the discrepancy and resolution of the discrepancy are discussed in the following two sections.

\subsubsection{Effect of Unconjugated mAb}

A possible source for the discrepancy is the presence of unlabeled $\mathrm{mAb}$ in the labeling solution (the labeling solution supplied by the manufacturer may contain unlabeled mAb). During labeling, both the labeled and unlabeled mAbs bind to the receptor sites on the surface of the cell. Assuming the two species have similar affinities for the receptor, subsequent to labeling, the cell surface will contain both $\mathrm{mAb}$ and labeled mAb. However, only labeled mAb will contribute to the MFI when the cell is analyzed using a flow cytometer. The concentration provided by the manufacturer, designated by con in the manuscript, gives the total concentration of the antibody and has to be separated into two parts con $=[$ labeled $m A b]+[m A b]$. The symbol [labeled $m A b$ ] gives the concentration of labeled $\mathrm{mAb}$, while $[m A b]$ is the concentration of unlabeled $\mathrm{mAb}$. The concentration [labeled $m A b$ ] can be represented by $f *$ con, where the fraction of labeled $\mathrm{mAb}$ is given by $f=\frac{[\text { labeled } m A b]}{\text { con }}$. The binding model uses $[A T+A T T]$ to describe the measured value of MFI on the vertical axis, and the total mAb concentration, con, on the horizontal axis. The concentration con gives a true representation of the saturation of binding, since both labeled and unlabeled $\mathrm{mAb}$ bind equally to the receptors on the cell surface. However, MFI depends only on the labeled portion of the bound mAb, given by $f \times([A T]+[A T T])$. Thus, the observed values of MFI are scaled-down versions of the true values when all of the mAb contributed to the fluorescence signal. To compensate for the unlabeled $\mathrm{mAb}$, the observed values of MFI should be scaled up by $1 / f$. Consequently, the value of the parameter $\left[T_{0}\right]$ obtained using the model to fit the observed values of MFI should be scaled up by $1 / f$ to include receptors bound to unlabeled $\mathrm{mAb}$. Assuming the fraction, $f$, of labeled $\mathrm{mAb}$ in the labeling solution was equal to 0.8 gave $65,000 / 0.8=81,000$ for the total number of CD4 receptors. This value is still too low. Taking $f=0.6$ increased the receptor number to 108,000 , which approaches the accepted value. The fraction of labeled $\mathrm{mAb}$ can be obtained by analyzing the labeling solution using size exclusion chromatography (SEC), which is generally used for purification of labeled mAb reagents. It is our understanding that it is extremely unlikely that two manufacturers provide labeling solutions of CD4 mAbAPC with $40 \%$ unlabeled mAb. The size difference between mAb $(150 \mathrm{kD})$ and $\mathrm{mAb}-\mathrm{APC}(267 \mathrm{kD})$ is sufficiently large for SEC to be a robust separation technique. 


\subsubsection{Analysis of CD4 mAb-APC Data Assuming Divalent Binding}

A better approach is to assume that the binding of CD4 mAb-APC to the CD4 receptor is divalent. Divalent binding was also invoked in the case of CD4 mAb-PE, where PE stands for phycoerythrin [11], which is a fluorescing protein similar to APC. The result of the model with divalent binding $\left(K_{1} \gg K_{2}\right)$ is shown in Fig. 3(d) for CD4 mAb-APC from company 2, and the parameter values that give the best fit are given in row 7 of Table 3 . Of greatest interest is the value of $\left[T_{0}\right]$, which in the divalent binding case is $0.527 \mathrm{nmol} / \mathrm{L}$. Thus, the predicted total number of CD4 receptors is $158,000 \pm 9000$. Similar analysis was performed for CD4 mAb-APC from company 1, with the fit results shown in Fig. 3(c) and the best-fit parameter values shown in row 5 of Table 3 . In the case of CD4 mAb-APC from company 1, the total number of CD4 receptors was found to be 139,000 \pm 3000 . The number of CD4 receptors found for the two CD4 mAb-APC conjugates is within two standard deviations of the value 145,000 \pm 9000 reported previously $[9,10]$. The difference in the values of the parameter $\left[T_{0}\right]$ between mAb labeled with FITC, about 114,000 , and those labeled with APC, about 147,000, is significant and may point to problems with producing a "pure" solution of CD4 mAb-FITC conjugates due to the small size difference between CD4 $\mathrm{mAb}(150 \mathrm{kD})$ and CD4 mAb-FITC $(150.4 \mathrm{kD})$. A fraction of 0.1 of unlabeled mAb in the CD4 mAb-FITC labeling solution would reconcile the difference in the values of $\left[T_{0}\right]$ for the FITC and APC CD4 mAb conjugates. The dominance of divalent binding in the case of CD4 mAb-APC and monovalent binding in the case of CD4 mAb-FITC is not understood at present.

In summary, Table 3 gives the parameters that gave the best fit to the measured binding curves and a number of CD4 receptors consistent with the known value. The parameter $\left[T_{0}\right]$, given in the fifth column of Table 3, was used in Eq. (3) (the relation between the number of bound species on cells and the effective volume concentration in the cell suspension) to obtain the total number of CD4 receptors shown in column 9. The average number of CD4 receptors on a lymphocyte was about 132,000 $\pm 11,000$ (average of column 9 in Table 3). This value is consistent with the known number of CD4 receptors, 145,000 $\pm 9000[9,10]$. The large uncertainties in the values of $K_{1}$ and $K_{2}$ suggest that the residue function has small curvatures in the $K_{1}$ and $K_{2}$ directions in parameter space. However, it is likely that the relation $K_{1}<<K_{2}$ holds for the pair of mAb labeled with FITC, and $K_{1} \gg K_{2}$ holds for the pair labeled with APC. This result suggests that the binding of labeled CD4 mAb to CD4 receptors is influenced by the size of the label attached to the $\mathrm{mAb}$. The model prediction of "label gain" (MFI/mAb) is similar for the three labels in rows 1, 3, and 5 in Table 3 and is about two times larger for the label in row 7. The measurements also give an estimate of the labeling quality index, defined as the difference between the highest MFI and the background, divided by two times the standard deviation (SD) of the background. The values of the labeling quality index have been reported previously [12]. Below is a discussion of the use of the model to provide additional characterization of the labeled mAb provided by different manufacturers.

\subsection{Comparison of Labeled CD4 mAb from Different Manufacturers}

At present, there is no accepted cell with a "standard" CD4 receptor and a "standard" CD4 mAb for the receptor. The best we can do is to compare CD4 mAbs with the same clonality from different manufacturers labeled with the same fluorophore. For example, it is possible to compare the two CD4 mAb-FITC conjugates obtained from company 1 and company 2, and the two CD4 mAb-APC conjugates from the same two companies. The comparison is possible because the cells labeled with the two similar conjugates are measured in the same fluorescence channel of the flow cytometer with identical instrument settings. Thus, all of the properties associated with the instrument in Eq. (9) cancel out, and we are left with the ratio shown in Eq. (10). It is assumed that the photon absorption cross section is the same for both labeled mAbs. 


$$
\frac{\left(\frac{M F I}{N_{a b}}\right)_{2}}{\left(\frac{M F I}{N_{a b}}\right)_{1}}=\frac{\left((Q Y) F_{a b}\right)_{2}}{\left((Q Y) F_{a b}\right)_{1}} .
$$

The two terms on the left side of Eq. (10) are the label gains given in column 7 of Table 3 . We can use the numbers in Table 3 to write the ratio as shown below for the case of CD4 mAb-FITC provided by company 1 and company 2 .

$$
1.10=\frac{\left((Q Y) F_{a b}\right)_{C o 2}}{\left((Q Y) F_{a b}\right)_{C o 1}} .
$$

This result suggests that the fluorescence properties of the two labels are very similar. Performing the same calculation for the ratio of the two CD4 mAb-APC labels, we get

$$
2.15=\frac{\left((Q Y) F_{a b}\right)_{C o 2}}{\left((Q Y) F_{a b}\right)_{C o 1}}
$$

The ratio of the two mAb-APC labels comes out to 2.15. This suggests that there may be two APC fluorophores attached to each mAb from company 2 and only one APC fluorophore attached to the mAb from company 1 . Alternately, there could be a significant number of unlabeled $\mathrm{mAb}$ in the preparation supplied by company 1 . It is unlikely that $Q Y$ can differ by a factor of two. In any case, there is a difference in the CD4 mAb-APC preparations supplied by the two manufacturers. The right side of Eq. (10) can be evaluated using independent fluorescence spectroscopy measurements on solutions of labeled antibodies. A good match between the value of the right side of Eq. (10) (spectroscopy) and the left side (flow cytometer and modeling) would give additional credence to the model.

Equation (10) is the confluence of binding measurements, modeling, and fluorescence measurements. Assuming that the simple model discussed in Sec. 3.1 is correct, it gives some interesting predictions regarding the valence of mAb binding, estimate of label gain, and the relative efficacy of identical labels from different manufacturers. It is worthwhile to improve the model and bring more certainty to the predictions. Two such improvements are discussed in Sec. 5.

\section{Enhancements of the Antibody Binding Model}

The discussion in Sec. 4 suggests that the measurements are well described by the model developed in Sec. 3. However, the interpretation of the parameters requires the examination of several factors neglected in the simplified model discussed in Sec. 3. One of these factors is the binding of mAb to monocytes present in the PBMC sample. Preliminary measurements indicate that the monocyte concentration is about a third of the lymphocyte concentration, and the monocyte MFI at saturating staining conditions is about a fifth of the lymphocyte MFI. Although relatively small, the monocytes will affect the binding of $\mathrm{mAb}$ to lymphocytes. The magnitude of this effect is examined in Sec. 5.1. The second important omission in the simplified model is nonspecific adsorption/binding of $\mathrm{mAb}$ to the surface of the cell. This process is examined in Sec. 5.2 by adding an adsorption step (state B in Fig. 1) to the model discussed in Sec. 3. The combined effect of monocyte binding and nonspecific adsorption is not discussed at this time. 


\subsection{Labeling of Lymphocytes in the Presence of Monocytes}

It will be assumed that the binding of labeled CD4 mAb to CD4 receptors on monocytes is very similar to their binding on lymphocytes, so that the dissociation and association equilibrium constants for the lymphocytes can be applied to the binding of labeled CD4 mAb to CD4 receptors on monocytes. The symbol $\left[T_{\mathrm{mo}}\right]$ represents the initial concentration of CD4 receptors on monocyte cells in the labeling solution, and the symbol $\left[T_{\mathrm{m}}\right]$ represents the concentration of unbound receptors on monocytes at equilibrium. There will be two additional equations describing the equilibrium state on the monocytes.

$$
\begin{gathered}
{[A]\left[T_{m}\right]-K_{1}\left[A T_{m}\right]=0 .} \\
{\left[A T_{m}\right]\left[T_{m}\right]-K_{2}\left[A T T_{m}\right]=0 .}
\end{gathered}
$$

The new variables $\left[T_{\mathrm{m}}\right],\left[A T_{\mathrm{m}}\right]$, and $\left[A T T_{\mathrm{m}}\right]$ give the concentration of unbound CD4 receptors, and the monovalently and divalently bound labeled CD4 mAbs on the monocyte surface, respectively. The monocyte and lymphocyte binding reactions share the same labeled $\mathrm{mAb}$ in the labeling suspension. The conservation equations have to be modified to include the labeled CD4 mAb on the monocyte cells as shown below.

$$
\begin{gathered}
{\left[A_{0}\right]=[A]+[A T]+[A T T]+\left[A T_{m}\right]+\left[A T T_{m}\right] .} \\
{\left[T_{0}\right]=[T]+[A T]+2 *[A T T]} \\
{\left[T_{m 0}\right]=\left[T_{m}\right]+\left[A T_{m}\right]+2 *\left[A T T_{m}\right]}
\end{gathered}
$$

There are separate conservation equations for receptors on monocytes, Eq. (15), and lymphocytes, Eq. (14), because the two receptor populations are independent of each other. In summary, the model contains two new equations, Eq. (11) and Eq. (12), describing the equilibrium binding of labeled CD4 mAb to CD4 receptors on monocytes. The dissociation constants in Eq. (11) and Eq. (12) were assumed to be the same as those for the binding of labeled CD4 mAb to CD4 receptors on lymphocytes. Equation (15) is a new conservation equation for the number of CD4 receptors on the monocyte surface, and Equation (13) is a modified conservation equation for the number of labeled CD4 mAbs in the labeling suspension. Equation (14) is the original conservation equation for CD4 receptors on the surface of lymphocytes. The form of Eqs. (13), (14), and (15) emphasizes the fact that the binding of labeled CD4 mAb to CD4 receptors on monocytes and lymphocytes is independent of each other; however, they both share the same pool of labeled CD4 mAb in the labeling solution.

The results from the previous modeling of lymphocyte binding in Sec. 3.1 were used as initial conditions for the variables in the solution of the system of seven nonlinear equations describing the binding of labeled CD4 mAb to CD4 receptors on lymphocytes and monocytes. To improve the speed of convergence, the Jacobian matrix associated with the seven equations was provided to fsolve. Figure 4 shows the effect of monocytes on the binding of CD4 mAb to CD4 receptors on lymphocytes. 


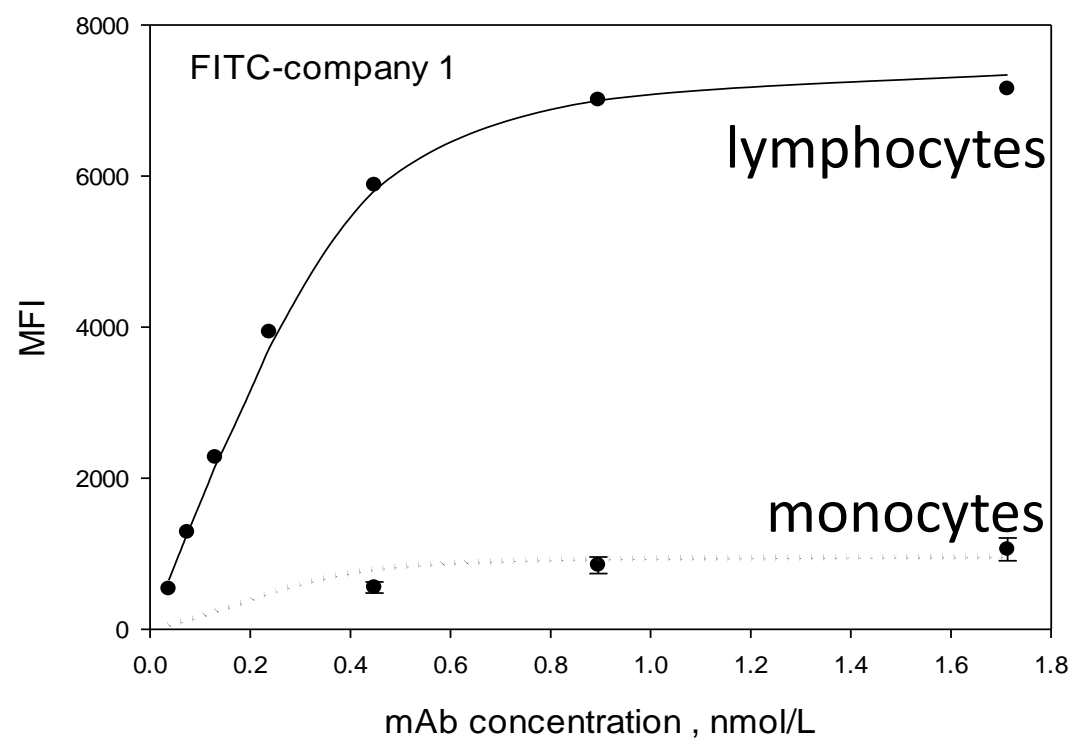

Fig. 4. The solid circles give the measured mean fluorescence intensity (MFI) associated with lymphocytes and monocytes labeled with solutions containing different concentrations of CD4 mAb-FITC conjugates provided by company 1 . The solid and dashed lines give the predictions of the model for MFI associated with lymphocytes and monocytes, respectively. The parameters used in the calculation are shown in first row in Table 3. The number of CD4 receptors on monocytes was 0.13 of the number of CD4 receptors on lymphocytes. The dissociation constants were the same for the two cell types. There was no attempt to get the best fit for the lymphocyte data.

The solid circles are measured MFI values for the CD4 mAb-FITC from company 1 taken from Fig. 3(a). The solid line is the model calculation for the case where $\left[T_{m o}\right]$ is $0.05 \mathrm{nmol} / \mathrm{L}$ and $\left[T_{0}\right]$ is $0.39 \mathrm{nmol} / \mathrm{L}$. The value for $\left[T_{m o}\right]$ was chosen so that the model reproduces the monocyte response shown by the solid circles in Fig. 4. The dotted line in Fig. 4 shows the calculated monocyte response. Using the gain parameter found for lymphocytes, the calculated monocyte signal at large values of $\left[A_{0}\right]$ is about 1200 on the MFI scale. The presence of monocytes has a small effect on the lymphocyte response at small CD4 $\mathrm{mAb}$ concentrations. At large CD4 mAb concentrations, the effect is insignificant. The fit of the calculated lymphocyte response to the data in Fig. 4 can be improved by performing a simultaneous fit to the lymphocyte and monocyte data. However, judging from the results shown in Fig. 4, the net improvement is expected to be small.

\subsection{Lymphocyte Binding Model with Inclusion of Nonspecific Adsorption}

The probability that a labeled CD4 mAb in solution will undergo a direct binding to a CD4 target receptor on a cell surface is very small. A more likely scenario is an association of the labeled CD4 mAb with the cell surface followed by a binding to the CD4 receptor. This scenario is shown in Fig. 1, where state B represents the labeled CD4 mAb associated with the cell surface. In what follows, the words association and adsorption will be used interchangeably. Equation (16) gives the equilibrium relation governing the concentration of labeled CD4 $\mathrm{mAb}$ associated with the cell surface.

$$
0=k_{\text {on }}[A] S-k_{\text {off }}\left[A_{S}\right]-k_{1}\left[A_{S}\right][T]+k_{-1}\left[A_{S} T\right] .
$$

Here, $S$ represents the cell surface area $\left(\mathrm{m}^{2}\right)$ per liter of suspension, $\left[A_{S}\right]$ is the concentration $(\mathrm{mol} / \mathrm{L})$ of labeled CD4 mAb associated with the cell surface, and $\left[\mathrm{A}_{S} \mathrm{~T}\right]$ is the concentration $(\mathrm{mol} / \mathrm{L})$ of labeled CD4 mAb bound to a CD4 receptor on the cell surface. As in previous discussion, Eq. (3) relates the volume 
concentration of any species to the number per cell of the same species. The constants $k_{\text {on }}$ and $k_{\text {off }}$ are the rate constants for adsorption and desorption of $\mathrm{mAb}$, respectively. The adsorption process can occur via a number of different interactions, e.g., interaction of charged groups, interaction of hydrophobic domains, and steric entanglement. It is assumed that all of the different interactions share the same cell geometry, so that the adsorption term in Eq. (16) can be written as a product of the cell surface area, the solution concentration of $\mathrm{mAb}$, and an absorption rate constant. It is also assumed that the total number of adsorption sites on the surface of the cell is very large and remains approximately constant during the labeling process (the fraction of available adsorptions sites remains close to 1). The cell surface, $S$, may have to be represented by a fractal object, and the absorption and desorption constants will have significant contribution from diffusion near a fractal surface [13]. The equilibrium equation for $\left[A_{\mathrm{S}}\right]$ is supplemented by additional equations for $\left[A_{\mathrm{S}} T\right]$, the population of labeled $\mathrm{CD} 4 \mathrm{mAb}$ bound to CD4 receptors monovalently, and $\left[A_{S} T T\right]$, the population of labeled CD4 mAb bound divalently to CD4 receptors. The concentrations at equilibrium are given by Eq. (17) and Eq. (18).

$$
\begin{gathered}
0=k_{+1}\left[A_{S}\right][T]-k_{-1}\left[A_{S} T\right]-k_{+2}\left[A_{S} T\right][T]+k_{-2}\left[A_{S} T T\right] . \\
0=k_{+2}\left[A_{S} T\right][T]-k_{-2}\left[A_{S} T T\right] .
\end{gathered}
$$

The two conservation equations become:

$$
\begin{gathered}
{\left[A_{0}\right]=[A]+\left[A_{S} T\right]+\left[A_{S} T T\right]+\left[A_{S}\right] .} \\
{\left[T_{0}\right]=[T]+\left[A_{S} T\right]+2 *\left[A_{S} T T\right] .}
\end{gathered}
$$

There are five equations for the five unknowns $[A],\left[A_{\mathrm{S}}\right],\left[A_{\mathrm{S}} T\right],\left[A_{\mathrm{S}} T T\right]$, and $[T]$. The model explicitly states that the binding of labeled CD4 mAb to CD4 receptors occurs after the adsorption of labeled CD4 $\mathrm{mAb}$ to the cell surface. The direct binding of labeled CD4 mAb in solution to CD4 receptors on the surface is neglected. The model given by Eq. (16) through Eq. (20) implements the full state diagram shown in Fig. 1. Adsorption is governed by a weak interaction, and since the preparation of the sample for flow cytometer measurement involves a separation and resuspension step, most of the adsorbed labeled CD4 mAb would be eliminated from the cell surface and not interfere with the fluorescence measurement from labeled CD4 mAb bound to the CD4 receptors on lymphocytes. Since the binding is a sequential process, the flux of reactants at each step must be conserved. Thus, Eq. (16) through Eq. (20) can be recast in a more compact form, shown below.

$$
\begin{aligned}
& 0=k_{1}\left[A_{S}\right][T]-k_{-1}\left[A_{S} T\right]=\left[A_{S}\right][T]-K_{1}\left[A_{S} T\right] . \\
& 0=k_{2}\left[A_{S} T\right][T]-k_{-2}\left[A_{S} T T\right]=\left[A_{S} T\right][T]-K_{2}\left[A_{S} T T\right] . \\
& 0=k_{\text {on }}[A] S-k_{\text {off }}\left[A_{S}\right]=[A] S-K_{D}\left[A_{S}\right] . \\
& {\left[A_{0}\right]=[A]+\left[A_{S} T\right]+\left[A_{S} T T\right]+\left[A_{S}\right] .} \\
& {\left[T_{0}\right]=[T]+\left[A_{S} T\right]+2 *\left[A_{S} T T\right] .}
\end{aligned}
$$

The model, given by the five equations above, was solved using the fsolve function in Matlab. As in Sec. 4.2, the solution in Sec. 3.1 provided initial values for the variables, and the Jacobian matrix was used to facilitate convergence. The solid line in Fig. 5 shows the calculated response, and the solid circles give the measured MFI for CD4 mAb-FITC shown in Fig. 3(a). 


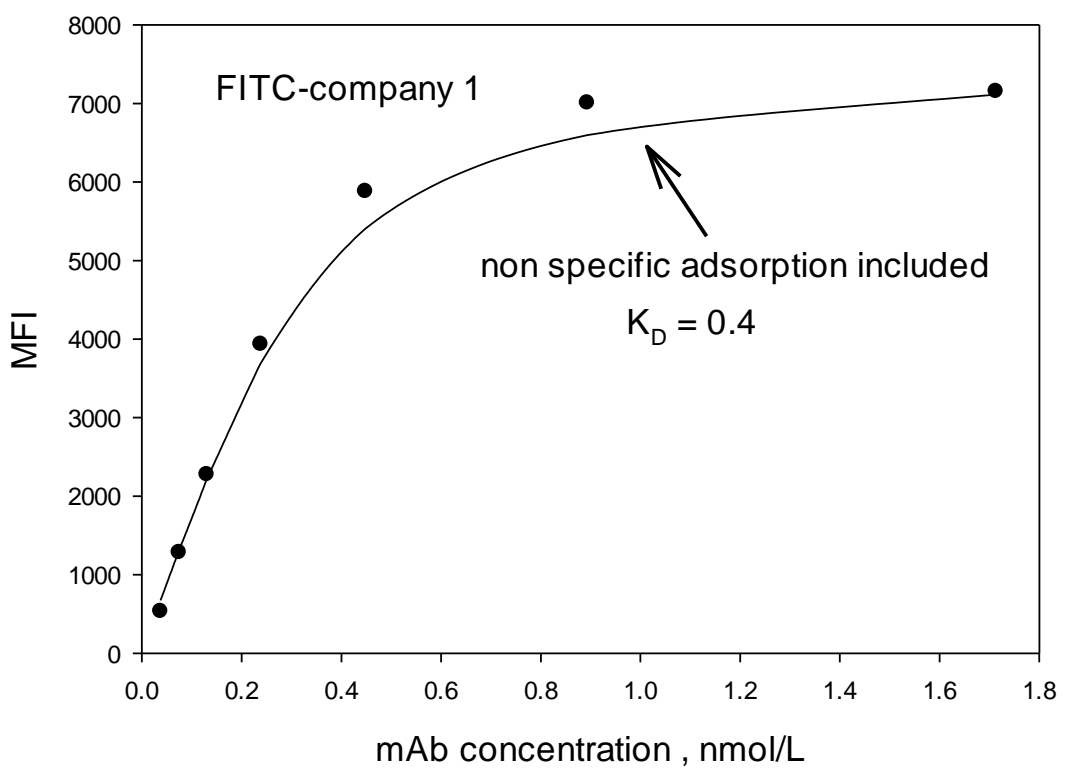

Fig. 5. The solid circles give the measured mean fluorescence intensity (MFI) associated with peripheral blood mononuclear cells (PBMC) labeled with solutions containing different concentrations of CD4 mAb-FITC conjugates provided by company 1 . The solid line gives the predictions of the model for MFI of lymphocytes when nonspecific adsorption is included in the model (state B in Fig. 1). The parameters used in the calculation are shown in the first row in Table 3 . The PBMC area was set to $0.084 \mathrm{~m}^{2} / \mathrm{L}$, and the ratio of the mAb desorption and adsorption rates was set to 0.4 . It is very likely that the ratio of desorption and adsorption rates is several orders smaller than 0.4. In that case, adsorption/desorption has minimal effect on the shape of the binding curve as modeled in Sec. 4.2 .

The parameters shown in row 1 of Table 3 were used in the calculation. The cell area was taken as $0.084 \mathrm{~m}^{2} / \mathrm{L}$ (for $0.2 \times 10^{7}$ cells $/ \mathrm{mL}$ and cell diameter of $7.3 \times 10^{-6} \mathrm{~m} \mathrm{[14]}$ ), and $K_{D}=k_{\text {off }} / k_{\text {on }}$ was set to 0.4. For such a large value of $K_{D}$, nonspecific adsorption may have a significant effect on the lymphocyte response, as indicated by the solid line in Fig. 5, and the fit parameters $G, K_{1}, K_{2}$, and [ $\left.T_{0}\right]$ will change when nonspecific adsorption is included in the model. However, the value of $K_{D}$ is expected to be much smaller $(<<0.1)[15,16]$, with negligible effect on the prediction of the model described in Sec. 3.1. In that case, the parameters $K_{1}$ and $K_{2}$ are associated with surface reaction between the adsorbed labeled CD4 mAb and the surface-bound CD4 receptors. There is a need for additional information to guide the choice of the value of the diffusion parameter, $K_{D}$, for CD4 mAb-dye conjugates.

\section{Conclusion}

A model was developed to describe the flow cytometer measurement of the equilibrium binding between labeled CD4 mAb and PBMCs with CD4 receptors. The cells were incubated in suspensions containing specified concentrations of labeled CD4 mAb. After a sufficient incubation time, the cells were resuspended in a PBS buffer and analyzed using a flow cytometer. The flow cytometer measured the mean fluorescence intensity (MFI) from the cell population. The MFI was a measure of the average number of bound labeled CD4 mAb on the cell surface. Equation (9) was used to relate the MFI to the predicted number of bound labeled CD4 mAbs on a cell. Model parameters were adjusted to give a good fit between the measured MFI values and the model predictions. In addition to a good fit, it was necessary to invoke divalent binding for CD4 mAb labeled with APC, and monovalent binding for CD4 mAb labeled with FITC in order to get a total number of $\mathrm{CD} 4$ receptors as close as possible to the previously measured value of $145,000 \pm 9000[9,10]$. As a result, the number of antibodies bound per cell (ABC) was two times 
higher for the CD4 mAb with FITC labels compared to CD4 mAb with APC labels. For any two preparations of $\mathrm{CD} 4 \mathrm{mAb}$ labeled with the same fluorophore, the ratio of their effective $F / P$ numbers (Table 1) was equal to the ratio of label gain, defined as the ratio of the MFI divided by the number of bound labeled CD4 mAbs (Eq. (10)). The ratio of label gains and the ratio of the effective $F / P$ for CD4 mAb-FITC from company 1 and company 2 were approximately one. For CD4 mAb APC conjugates, the ratio of the label gains was about 2.1, but the ratio of the effective $F / P$ values was close to 1 . These results will be reconciled in the future using fluorescence spectroscopy measurements on the solutions of labeled $\mathrm{CD} 4 \mathrm{mAb}$. The model was extended to include the competitive binding of labeled CD4 mAb to monocytes. For low concentrations of labeled CD4 mAb, the presence of monocytes leads to small observable effects on the equilibrium binding of labeled mAb to CD4 receptors on lymphocytes. For large concentrations of CD4 mAb, the presence of monocytes had minimal effect. The model was also extended to include nonspecific adsorption of labeled CD4 mAb on lymphocytes. Preliminary calculations indicate that nonspecific adsorption most likely does not change the shape of the CD4 mAb binding curve. It is likely that the binding of labeled CD4 mAb is a surface reaction between the adsorbed labeled CD4 mAb and the CD4 receptors on the surface of the cell. It is anticipated that the reconciliation of flow cytometer measurements, the predictions of binding models, and fluorescence spectroscopy measurements will be an important step in the quest for quantitative flow cytometry measurements.

\section{Appendix}

Four separate functions were used for the analysis of the binding data of labeled $\mathrm{mAb}$ to receptors on the surface of lymphocytes. First, the model equations described in Sec. 3.1 of the manuscript were implemented in the Matlab function lymph $\left(v\right.$, par, $A_{0}$ ). The function lymph specifies (does not solve) the model equations for a set of parameters contained in the variables par and $A_{0}$, and the concentration variables contained in $v$. Second, the function FIlymph(par,con,MFI,err,save) evaluates the model equations provided in lymph for a set of $\mathrm{mAb}$ concentrations given in the variable con, and the parameters specified in par. The function FIlymph evaluates the model equations for each concentration in the vector variable con using the same set of parameters in par. The function fitdata2 (par,con,MFI,err,SD) uses the initial guess values of the parameters given in par to find the best representation of the measured binding curve specified by the variables con and MFI. The best fit is found using the Matlab algorithm lsqnonlin, which searches for a minimum of the function given in Eq. (A1).

$$
\operatorname{residue}^{2}(p)=\sum_{i=1}^{m}\left(M F I_{i}-\left(p_{4} \operatorname{FIlymph}\left(p, \operatorname{con}_{i}, M F I_{i}, \text { err, } 0\right)+p_{5}\right)\right)^{2} .
$$

The index $i$ runs over the entire range of measured values of concentration and MFI. (The index $i$ is used in Eq. (A1) to clarify the procedure. In practice, Eq. (A1) is written compactly using Matlab matrix notation.) The solver lsqnonlint permits a selection of algorithms that search for a minimum, and it calculates the Jacobian matrix at the minimum. The Jacobian matrix is used to calculate the uncertainties in the values of the parameters that give the best fit. Isqnonlin requires the input of a residue vector. An example is shown in Table A1 for the case of a binding curve with seven measured pairs of concentration and MFI.

Table A1. Residue vector for the case of a binding curve with seven measured pairs of concentration and MFI.

\begin{tabular}{|c|}
\hline$\left(M F I_{1}-p_{4} * F I\left(\operatorname{con}_{1}, p\right)-p_{5}\right)$ \\
\hline$\left(M F I_{2}-p_{4} * F I\left(\operatorname{con}_{2}, p\right)-p_{5}\right)$ \\
\hline$\left(M F I_{3}-p_{4} * F I\left(\operatorname{con}_{3}, p\right)-p_{5}\right)$ \\
\hline$\left(\mathrm{MFI}_{4}-p_{4} * F I\left(\mathrm{con}_{4}, p\right)-p_{5}\right)$ \\
\hline$\left(M F I_{5}-p_{4} * F I\left(\operatorname{con}_{5}, p\right)-p_{5}\right)$ \\
\hline$\left(M F I_{6}-p_{4} * F I\left(\operatorname{con}_{6}, p\right)-p_{5}\right)$ \\
\hline$\left(M F I_{7}-p_{4} * F I\left(\operatorname{con}_{7}, p\right)-p_{5}\right)$ \\
\hline
\end{tabular}


Each component of the residue vector is the difference between the measured value of MFI and the prediction of MFI for the mAb concentrations used in the binding study. The parameter $p_{4}$ is the gain, $p_{5}$ is the background, $p_{1}$ is $K_{1}, p_{2}$ is $K_{2}$, and $p_{3}$ is [ $\left.T_{0}\right]$. The function FIlymph calculates the components of the residue vector prior to calling lsqnonlin, which squares the components of the residue vector and proceeds with the search for the minimum. The output of 1sqnonlin consists of the parameters that yield the minimum of the residue vector magnitude, the final residue vector, and the Jacobian matrix evaluated at the minimum.

The uncertainties in the parameters, $\sigma_{j}^{2}$, were calculated using the formula presented on page 147 in Ref. [17], which is rewritten as Eq. (A2).

$$
\sigma_{j}^{2}=\frac{2}{\frac{\partial^{2} \chi}{\partial p_{j}^{2}}}
$$

The $\chi^{2}$ is a modified form of Eq. (A1), where each squared term in the sum is divided by the square of the standard deviation (SD) of the MFI. Thus, the square of the difference between the measured and predicted values is given in units of the square of the SD of the measured value. The $\chi^{2}$ becomes a measure of the quality of the fit, a property absent from the residue function shown in Eq. (A1). In addition, $\chi^{2}$ is divided by the difference between the number of observations and the number of parameters. Therefore, at the minimum, $\chi^{2}$, should be of the order of 1 , and it will increase quadratically with parameter deviation from the values at the minimum. The function $\chi^{2}$ is used to justify Eq. (A2), as demonstrated in the following paragraph.

The second derivative of $\chi^{2}$ can be estimated by first expanding $\chi^{2}$ as a function of $\left(p_{j}-p_{j}^{*}\right)$, where $p_{j}^{*}$ is the value of the $j$ th parameter at the minimum. It will be assumed that a first-order expansion will be sufficient. In that case, the expansion is written as shown in Eq. (A3).

$$
\chi^{2}(p)=\sum_{i=1}^{m}\left(\left(M F I_{i}-f\left(\operatorname{con}_{i}, p^{*}\right)-\sum_{j=1}^{5} \frac{\partial f\left(\operatorname{con}_{i}, p^{*}\right)}{\partial p_{j}}\left(p_{j}-p_{j}^{*}\right)\right)^{2} \frac{1}{\sigma_{i}^{2}}\right.
$$

The symbol $f\left(\mathrm{Con}_{i}, p^{*}\right)$ stands for everything that is subtracted from MFI in Eq. (A1). The Jacobian matrix at the minimum is defined in Eq. (A4).

$$
\left.J_{i, j}=\frac{1}{\sigma_{i}^{2}} \frac{\partial f\left(\operatorname{con}_{\mathrm{I}} \mathrm{p}\right)}{\partial p_{j}}\right]_{p_{j}^{*}} .
$$

Equation (A4) can be used in Eq. (A3) to represent the derivatives of $f(c o n, p)$. Next, we expand the squared term in Eq. (A3) and note that at the minimum of $\chi^{2}$, the first derivative of $\chi^{2}$ with respect to $p_{\mathrm{i}}$ is equal to zero. Note that the derivatives of $f(c o n, p)$ with respect to $p$ are not necessarily zero at the minimum. After some rearrangement, Eq. (A3) simplifies to the result given in Eq. (A5).

$$
\chi^{2}(p)-\chi^{2}\left(p^{*}\right)=\sum_{\mathrm{j}, \mathrm{k}=1}^{5}\left(\mathrm{~J}^{\mathrm{T}} \mathrm{J}\right)_{\mathrm{j}, \mathrm{k}}\left(p_{j}-p_{j}^{*}\right)\left(p_{k}-p_{k}^{*}\right) .
$$

Equation (A5) can be used to estimate the second derivative of $\chi^{2}$ at the minimum. After some algebra, the second derivative evaluates to the result shown in Eq. (A6).

$$
\frac{\partial^{2} \chi}{\partial p_{j}^{2}}=2\left(J^{T} J\right)_{j, j} .
$$

Equation (A6) is used in Eq. (A2) to provide an estimate of uncertainty in the values of the parameters shown in Eq. (A7). 


$$
\sigma_{j}^{2}=\frac{2}{2\left(J^{T} J\right)_{j, j}}=\frac{1}{\left(J^{T} J\right)_{j, j}}
$$

Equation (A5) provides a perspective on the uncertainty formula. The increase by one unit in the difference $\chi^{2}(p)-\chi^{2}\left(p^{*}\right)$ is a measure of parameter excursion, which still gives a reasonable fit. Therefore, Eq. (A8)

$$
\chi^{2}(p)-\chi^{2}\left(p^{*}\right)=1=\sum_{\mathrm{j}, \mathrm{k}=1}^{5}\left(\mathrm{~J}^{\mathrm{T}} \mathrm{J}\right)_{\mathrm{j}, \mathrm{k}}\left(p_{j}-p_{j}^{*}\right)\left(p_{k}-p_{k}^{*}\right)
$$

can be solved for a given parameter deviation from minimum while other parameters are held at their value at the minimum. This gives the range of parameter values that still gives a reasonable fit. The range is equal to that given by the uncertainty formula in Eq. (A2). The procedure is carried out for each parameter, leading to an estimate of error. Clearly, the uncertainties given by Eq. (A2) do not include effects due to correlations between parameters. Nevertheless Eq. (A7) is a practical estimate of the uncertainty. However, Eq. (A7) has to be modified because the Jacobian matrix calculated by lsqnonlin is given by Eq. (A9) and lacks $\sigma_{i}^{2}$ in the denominator.

$$
\left.J_{i, j}=\frac{\partial f\left(\operatorname{con}_{\mathrm{I}}, \mathrm{p}\right)}{\partial p_{j}}\right]_{p_{j}^{*}}
$$

The modification that is adopted here is to multiply Eq. (A9) by $1 /\left\langle\right.$ residues $\left.^{2}\right\rangle$, where $\left\langle\operatorname{residues}^{2}\right\rangle$ is the average value of residues squared for all data points. The final form of the uncertainty estimate is given in Eq. (A10).

$$
\sigma_{j}^{2}=\frac{\sum \text { residues }^{2}}{m-n} \frac{1}{\left(J^{T} J\right)_{j, j}} .
$$

Here, $m$ is the number of data points ( 7 to 8$)$, and $n$ is the number of parameters $(n=5)$. Expressions for uncertainties that include correlation effects have been presented on page 155 in [18]. The uncertainties are obtained by inverting Eq. (A8). Future analyses will include this improvement. This manuscript used uncorrelated uncertainties calculated using a modified form of Eq. (A2) given by Eq. (A10).

The Matlab code for the analysis of the data in this manuscript can be obtained from paul.derose@nist.gov.

Online references for appendix: http://www2.compute.dtu.dk/ pcha/LSDF/NonlinDataFit.pdf http://www2.imm.dtu.dk/pubdb/views/edoc_download.php/3215/pdf/imm3215.pdf.

\section{References}

[1] Wang L, Degheidy H, Abbasi F, Mostowski H, Marti G, Bauer S, Hoffman RA, Gaigalas AK (2016) Quantitative flow cytometry measurements in antibodies bound per cell based on a CD4 reference. Current Protocols in Cytometry 75:1.29.11.29.14. https://doi.org/10.1002/0471142956.cy0129s75

[2] Kantor AB, Moore WA, Meehan S, Parks DR (2016) A quantitative method for comparing the brightness of antibody-dye reagents and estimating antibodies bound per cell. Current Protocols in Cytometry 77:1.30.1-1.30.23. https://doi.org/10.1002/cpcy.6

[3] Wang L, Gaigalas AK, Wood J (2018) Quantitative fluorescence measurements with multicolor flow cytometry. Flow Cytometry Protocols, eds Hawley TS, Hawley RG (Springer Science + Business Media, LLC, New York), 4th Ed., pp 93-110.

[4] Wang L, Gaigalas AK (2011) Development of multicolor flow cytometry calibration standards: Assignment of equivalent reference fluorophores (ERF) unit. Journal of Research of the National Institute of Standards and Technology 116(3):671-683. https://doi.org/10.6028/jres.116.012

[5] Kaufman EN, Jain RK (1992) Effect of bivalent interaction upon apparent antibody affinity: Experimental confirmation of theory using fluorescence photobleaching and implications for antibody binding assays. Cancer Research 52(15):4157-4167. Available at: http://cancerres.aacrjournals.org/content/52/15/4157 
[6] Chen J, Almo SC, Wu Y (2017) General principles of binding between cell surface receptors and multi-specific ligands: A computational study. PLoS Computational Biology 13(10):e1005805. https://doi.org/10.1371/journal.pcbi.1005805

[7] Rhoden JJ, Dyas GL, Wroblewski VJ (2016) A modeling and experimental investigation of the effects of antigen density, binding affinity, and antigen expression ratio on bispecific binding to cell surface targets. The Journal of Biological Chemistry 291:11337-11347. https://doi.org/10.1074/jbc.M116.714287

[8] Vauquelin G, Charlton SJ (2013) Exploring avidity: Understanding the potential gains in functional affinity and target residence time of bivalent and heterobivalent ligands. British Journal of Pharmacology 168(8):1771-1785. https://doi.org/10.1111/bph.12106

[9] Siiman O, Burshteyn A (2000) Cell surface receptor-antibody association constants and enumeration of receptor sites for monoclonal antibodies. Cytometry 40(4):316-326. https://doi.org/10.1002/1097-0320(20000801)40:4<316::AIDCYTO7>3.0.CO;2-C

[10] Wang M, Misakian M, He H-J, Bajcsy P, Abbasi F, Davis JM, Cole KD, Turko IV, Wang L (2014) Quantifying CD4 receptor protein in two human CD4+ lymphocyte preparations for quantitative flow cytometry. Clinical Proteomics 11:43. https://doi.org/10.1186/1559-0275-11-43

[11] Davis KA, Abrams B, Iyer S, Hoffman R, Bishop J (1998) Determination of CD4 antigen density on cells. Role of antibody valency, avidity, clones, and conjugation. Cytometry 33(2):197-205. https://doi.org/10.1002/(SICI)10970320(19981001)33:2<197::AID-CYTO14>3.0.CO;2-P

[12] Wang L, Gaigalas AK, He H-J, Stein EV, DeRose PC (2017) Fluorescently labeled monoclonal metrology for quantitative flow cytometry. CYTO 2017, poster number 279.

[13] Dewey TG (1997) Fractals in Molecular Biophysics, ed Truhlar DG (Oxford, UK, Oxford University Press).

[14] Kuse R, Schuster S, Schnubbe H, Dix D, Hausmann K (1985) Blood lymphocyte volumes and diameters in patients with chronic lymphocytic leukemia and normal controls. Blut 50(4):243-248. https://doi.org/10.1007/BF00320301

[15] Dee KC, Puleo DA, Bizios R (2002) An Introduction to Tissue-Biomaterial Interactions (Wiley, New York), p 44. https://doi.org/10.1002/0471270598

[16] Pan C (2011) Measuring dissociation rate constants of protein complexes through subunit exchange: Experimental design and theoretical modeling. PLOS ONE 6(12):1-5, e28827. https://doi.org/10.1371/journal.pone.0028827

[17] Bevington PR, Robinson DK (2003) Data Reduction and Error Analysis for the Physical Sciences (McGraw Hill, New York), 3rd Ed.

[18] Hansen PC, Pereyra V, Scherer G (2012) Least Squares Data Fitting with Applications (Johns Hopkins University Press, Baltimore, MD).

About the authors: Lili Wang is a research chemist in the Biosystems and Biomaterials Division of the Materials Measurement Laboratory at NIST. She carries out measurements of clinically relevant biological substances, cells, proteins, DNA and RNA using quantitative flow cytometry.

Adolfas K. Gaigalas is a contractor in the Biosystems and Biomaterials Division at NIST. He carries out research on the standardization of fluorescence-based flow cytometer assays.

Paul C. DeRose is a research chemist in the Biosystems and Biomaterials Division at NIST. He carries out research on quantitative fluorescence and absorption spectrometry.

The National Institute of Standards and Technology is an agency of the U.S. Department of Commerce. 